\title{
INTRUSIÓN DE CERCLAJE ESCLERAL 19 AÑOS DESPUÉS
}

\section{A SCLERAL BUCLE INTRUSION 19 YEARS AFTER ITS USE IN RETINAL DETACHMENT SURGERY}

\author{
GARAY-ARAMBURU $\mathrm{G}^{1}$, LARRAURI-ARANA A ${ }^{1}$
}

\section{RESUMEN}

Caso clínico: Se define intrusión como la erosión y protrusión del implante escleral en la cavidad vítrea. Esta condición puede presentarse como desprendimiento de retina, hemorragias vítreas, endoftalmitis o de manera asintomática. Realizamos vitrectomía a una paciente con hemorragias vítreas de repetición secundarias a intrusión del cerclaje escleral implantado 19 años antes y dejamos intacto el cerclaje intraocular.

Discusión: La intrusión del cerclaje escleral es una complicación infrecuente de la cirugía del desprendimiento de retina. Discutimos el manejo de esta patología.

Palabras clave: Desprendimiento de retina, hemorragia vítrea, implante, intrusión cerclaje escleral, vitrectomía.

\begin{abstract}
Case report: Intrusion is defined as erosion and protrusion of the scleral implant into the vitreous cavity. This condition may occur as a retinal detachment, vitreous hemorrhage, endophthalmitis or be without symptoms. We performed a vitrectomy alone in a patient with relapsing vitreous hemorrhage secondary to the intrusion of a scleral buckle implanted 19 years previously and left intact the intruding buckle.

Discussion: Intrusion of the scleral buckle is an uncommon complication of retinal detachment surgery. We discuss the management of scleral buckle intrusion (Arch Soc Esp Oftalmol 2007; 82: 443446).
\end{abstract}

Key words: Implant, retinal detachment, scleral buckle intrusion, vitrectomy, vitreous hemorrhage.

\section{INTRODUCCIÓN}

Se define intrusión como la erosión y protrusión del implante escleral en la cavidad vítrea (1). La intrusión puede ser causada tanto por los materiales implantados sobre la esclera como por los intraesclerales, incluidas las suturas (1-3).

Comunicamos el caso de una paciente con hemorragias vítreas $(\mathrm{HV})$ de repetición secundarias a la intrusión del cerclaje escleral tras 19 años de la cirugía.

\section{CASO CLÍNICO}

Se trata de paciente mujer de 65 años seguida regularmente en nuestro servicio tras haber sido intervenida en dos ocasiones de desprendimiento de retina

Recibido: 1/8/06. Aceptado: 12/6/07.

Servicio de Oftalmología. Hospital de Txagorritxu de Vitoria. Vitoria. España.

1 Licenciado en Medicina.

Correspondencia:

Gonzaga Garay Aramburu

Servicio de Oftalmología. Hospital de Txagorritxu

José Atxotegi, s/n

01009 Vitoria

España

E-mail: ggaray@htxa.osakidetza.net 
(DR) en su ojo derecho (OD) en 1978. La primera cirugía consistió en diatermia en cuadrante nasal superior donde existía un desgarro retiniano y colocación de implante escleral segmentado. Al mes, tras sufrir una recidiva del DR, se le coloco cerclaje escleral completo anudado en cuadrante temporal inferior y se realizó diatermia en este cuadrante por existir degeneración retiniana. La paciente presentó un buen postoperatorio en el que se observó buena indentación del implante segmentado y cerclaje prominente.

A pesar de no tener síntomas, en 1997 la exploración reveló perforación escleral con intrusión del cerclaje de media hora de extensión en el área temporal inferior, en una zona extensa de atrofia coriorretiniana (fig. 1). Debido a la extensión de la intrusión y a la extensa zona de atrofia coriorretiniana se decidió la observación de la paciente.

Un año después, la paciente fue intervenida de catarata en ojo derecho mediante facoemulsificación e implante de lente en saco capsular. Siete años más tarde, se realizó capsulotomía YAG en OD por opacidad capsular. La paciente acudió a la consulta un mes después con HV en OD. La ecografía mostró la HV, la inexistencia de DR y la intrusión del cerclaje (fig. 2). Tras reabsorberse parcialmente la HV durante el primer mes, la paciente tuvo un nuevo sangrado por lo que se realizó vitrectomía para limpiar la sangre.

Dieciséis meses tras la cirugía, la paciente permanece asintomática y sin extensión del tamaño intraocular del cerclaje.

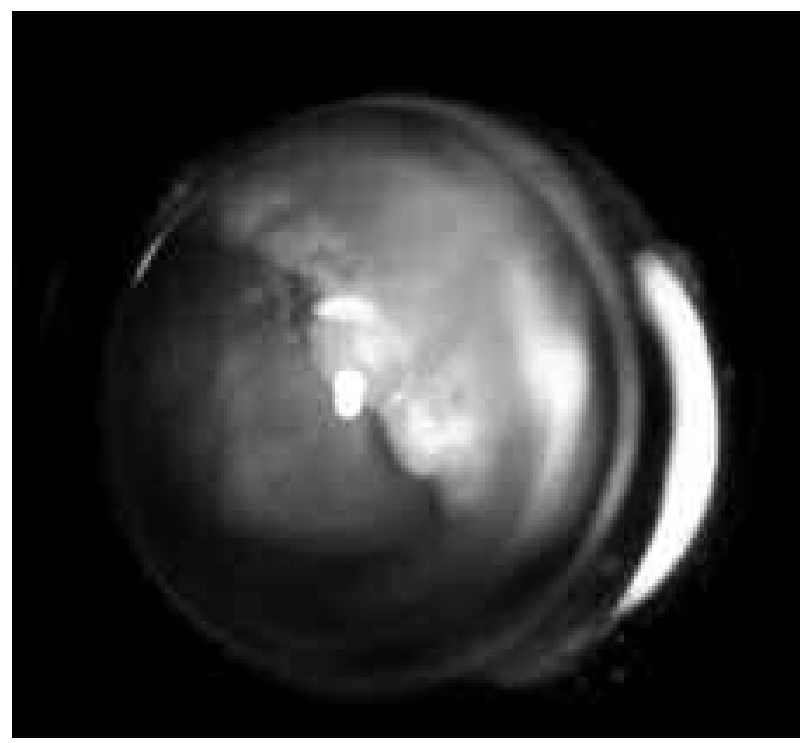

Fig. 1: Intrusión del cerclaje escleral.

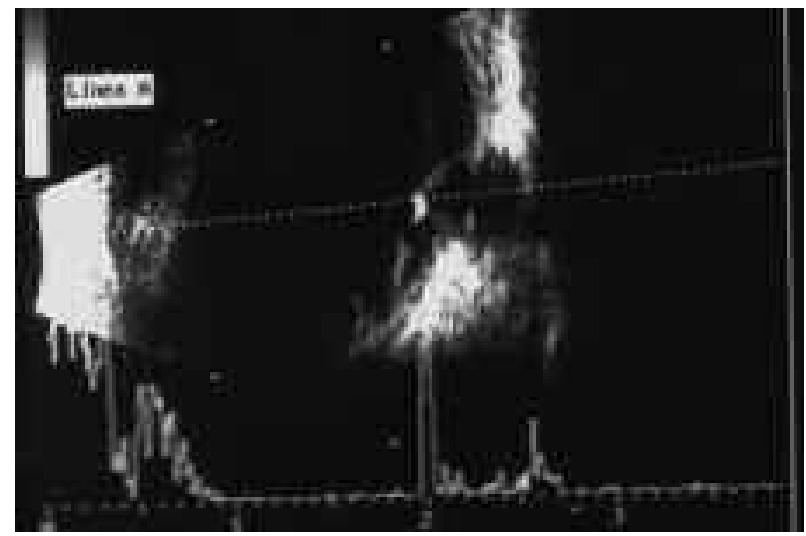

Fig. 2: Ecografía mostrando la intrusión.

\section{DISCUSIÓN}

La intrusión del cerclaje escleral es una complicación infrecuente de la cirugía del DR, se observa en 4 de cada 4400 casos (1). Los factores que favorecen la intrusión son la miopía, el glaucoma, el cerclaje escleral tenso y el tratamiento intensivo de pexia (4). En nuestro caso la paciente reunía tres de estos factores de riesgo ya que era miope magna, se había realizado pexia intensa mediante diatermia en el cuadrante temporal inferior y presentaba el cerclaje tenso ademas de estar anudado en dicho cuadrante. Los miopes magnos presentan una esclera adelgazada que unido a la pexia intensa favorecen la intrusión de un cerclaje tenso.

Nuestro diagnostico fue un hallazgo casual, dado que la paciente no tenía síntomas, pero esta condición puede presentares como DR o HV recurrentes (como sucedió en nuestro caso a los siete años del diagnóstico) y también puede presentarse como un riesgo mayor para el ojo como es la endoftamitis (2).

El manejo de la intrusión depende de la extensión intraocular del cerclaje y de la clínica que presente el paciente. La extracción del cerclaje no es obligada. Se puede tener una actitud expectante o se puede relajar el cerclaje o extraerlo parcialmente (1). En el caso de existencia de DR, HV o de que exista serio peligro para la integridad ocular se puede realizar una vitrectomía. El defecto escleral se puede reparar con un parche de tejido escleral (1-3).

Debido al tamaño intraocular del cerclaje y a que la retina circundante estaba atrófica y adelgazada, se decidió junto con la paciente, una actitud expectante con el cerclaje intraocular. Consideramos por tanto que el tratamiento debe ser individualizado en 
función de la extensión intraocular del cerclaje, de la clínica y de los deseos del paciente.

\section{BIBLIOGRAFÍA}

1. Nguyen QD, Lashkari K, Hirose T, Pruett RC, McMeel $J W$, Schepens $C L$. Erosion and intrusion of silicone rub- ber scleral buckle. Presentation and management. Retina 2001; 21: 214-220.

2. Kumar N, Zeldovich A, Chang A. Scleral buckle intrusion. Clin Experiment Ophthalmol 2004; 32: 228-229.

3. Cooper BA, Blinder KJ, Shah GK, Boniuk I. Intruding scleral sutures 12 years after primary repair. Arch Ophthalmol 2004; 122: 1238-1239.

4. Birgul T, Vidic B, El-Shabrawi Y. Intrusion of an encircling bucle alter retinal detachment surgery. Am J Ophthalmol 2003; 136: 942-944. 